DOI:

10.1038/nphys 857

\title{
Answers on a postcard
}

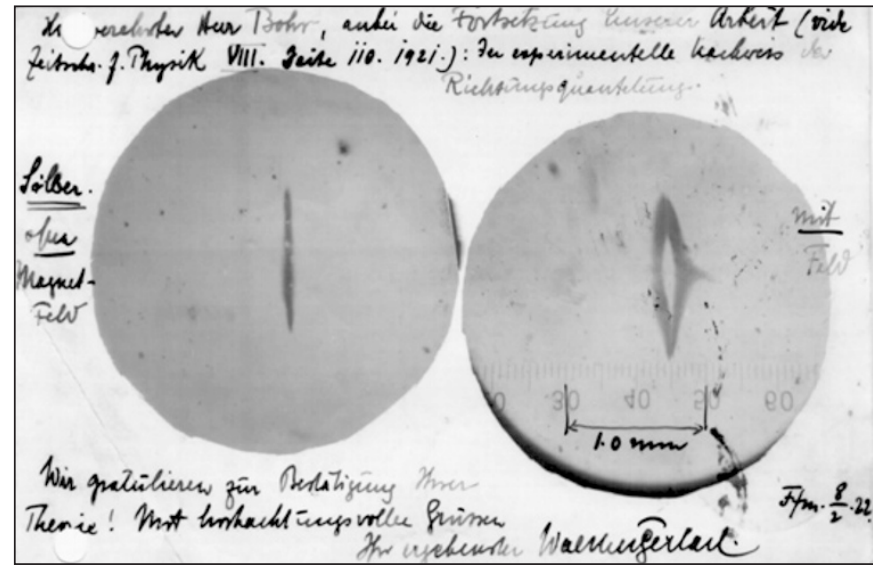

Postcard from Gerlach to Bohr. Image courtesy of Niels Bohr Archive, Copenhagen.
1922, Otto Stern and Walter Gerlach demonstrated such a reality beyond all reasonable doubt.

Their experiment involved passing a collimated beam of silver atoms through an inhomogeneous magnetic field and onto a glass slide where the deposits formed a pattern. Classical models suggested that the electron orbitals around the nucleus of these atoms should be randomly and continuously distributed, and that a single, broad and continuous spot of silver should form in the centre of the slide. The BohrSommerfeld model, by contrast, predicted that space quantization of these orbitals should cause the beam to be split into several discrete parts in the inhomogeneous field, forming discrete lines of silver deposits on the slide.

Despite its elegant simplicity, the experiment almost never happened. For one thing, for there to be any observable splitting, the alignment of the beam and the centre of the magnetic field had to be just right. More prosaically, in the middle of a worsening economic depression, funding the construction of the experiment proved to be almost as difficult. Thankfully, perseverance on the part of Stern and Gerlach, and a cheque for several hundred dollars provided by Henry Goldman (co-founder of the investment firm Goldman Sachs), allowed them to observe the splitting predicted by quantum theory - a result that is now most famously recorded on a postcard from Gerlach to Bohr congratulating him on the success of his theory.

Although the Stern-Gerlach experiment categorically disproved classical models of the atom, it was also inconsistent with the Bohr-Sommerfeld model. In fact, the observed splitting of the silver beam had nothing to do with the orbital angular momentum, but was due to the spin angular momentum of the unpaired electron in the atomic structure of silver - something that was not appreciated until years later, following the introduction of the idea of electron spin by Wolfgang Pauli (Milestone 3).

Not content with realizing what is perhaps the clearest and most direct demonstration of the quantum nature of atoms, Stern went on to demonstrate and measure the quantized spin of the proton - together with the size of its magnetic moment - for which he was awarded the 1943 Nobel Prize in Physics.

Ed Gerstner, Senior Editor, Nature Physics

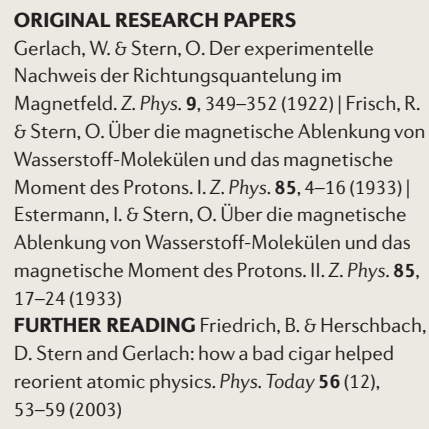

